\title{
On the Superstability of Lobačevskiir's Functional Equations with Involution
}

\author{
Jaeyoung Chung, ${ }^{1}$ Bogeun Lee, ${ }^{2}$ and Misuk $\mathrm{Ha}^{2}$ \\ ${ }^{1}$ Department of Mathematics, Kunsan National University, Kunsan 573-701, Republic of Korea \\ ${ }^{2}$ Department of Mathematics, Jeonbuk National University, Jeonju 561-756, Republic of Korea \\ Correspondence should be addressed to Bogeun Lee; akiles@jbnu.ac.kr
}

Received 29 November 2015; Accepted 22 February 2016

Academic Editor: Alberto Fiorenza

Copyright (c) 2016 Jaeyoung Chung et al. This is an open access article distributed under the Creative Commons Attribution License, which permits unrestricted use, distribution, and reproduction in any medium, provided the original work is properly cited.

Let $G$ be a uniquely 2-divisible commutative group and let $f, g: G \rightarrow \mathbb{C}$ and $\sigma: G \rightarrow G$ be an involution. In this paper, generalizing the superstability of Lobačevskiřs functional equation, we consider $\left|f((x+\sigma y) / 2)^{2}-g(x) f(y)\right| \leq \psi(x)$ or $\psi(y)$ for all $x, y \in G$, where $\psi: G \rightarrow \mathbb{R}^{+}$. As a direct consequence, we find a weaker condition for the functions $f$ satisfying the Lobačevskil functional inequality to be unbounded, which refines the result of Găvrută and shows the behaviors of bounded functions satisfying the inequality. We also give various examples with explicit involutions on Euclidean space.

\section{Main Results}

Throughout this paper, we denote by $G$ a uniquely 2-divisible commutative group (i.e., for each $x \in G$, there exists a unique $y \in G$ such that $y+y=x$ ) and by $\mathbb{R}, \mathbb{R}^{+}, \mathbb{C}$, and $\mathbb{R}^{n}$ the set of real numbers, nonnegative real numbers, complex numbers, and the $n$-dimensional Euclidean space, respectively. A function $m: G \rightarrow \mathbb{C}$ is called an exponential function provided that $m(x+y)=m(x) m(y)$ for all $x, y \in G$ and $\sigma: G \rightarrow G$ is called an involution provided that $\sigma(x+y)=$ $\sigma(x)+\sigma(y)$ and $\sigma(\sigma(x))=x$ for all $x, y \in G$. We denote $\sigma(x)$ by $\sigma x$. An exponential function $m: G \rightarrow \mathbb{C}$ is called $\sigma$-exponential function if $m$ satisfies $m(\sigma x)=m(x)$ for all $x \in G$ and is denoted by $m_{\sigma}$. In [1] (see also [2, Theorem 6.5]), Găvrută investigated the superstability of Lobačevskiǐ's functional equation:

$$
\left|f\left(\frac{x+y}{2}\right)^{2}-f(x) f(y)\right| \leq \delta
$$

for all $x, y \in G$ and for some $\delta>0$ (see Albert and Baker [3] and Baker [4] for the superstability of the exponential functional equation). As a result he proved the following.
Theorem 1. Assume that $f: G \rightarrow \mathbb{C}$ satisfies (1). Then, every bounded function $f$ satisfies

$$
|f(x)| \leq \frac{|f(0)|+\sqrt{|f(0)|^{2}+4 \delta}}{2}:=K_{f, \delta}
$$

for all $x \in G$, and if there exists $y_{0} \in G$ such that $\left|f\left(y_{0}\right)\right|>K_{f, \delta}$, then $f$ satisfies

$$
f\left(\frac{x+y}{2}\right)^{2}=f(x) f(y)
$$

for all $x, y \in G$.

A careful observation shows that the upper bound $K_{f, \delta}$ in (2) is not optimal and may be replaced by a better one; namely, $K_{f, \delta}^{*}=(1 / 2)\left(m_{f}+\sqrt{m_{f}^{2}+4 \delta}\right)$ with $m_{f}=\inf _{x \in G}|f(x)|$. For example, let $f(x)=\sqrt{\delta} \cos x$. Then, $K_{f, \delta}=(1 / 2)(1+$ $\sqrt{5}) \sqrt{\delta}$ and $K_{f, \delta}^{*}=\sqrt{\delta}$. Using the better upper bound, we investigate the behavior of bounded functions satisfying (1) (see Remark 3). 
As main results in this paper, we generalize Găvrută's result and consider the inequalities:

$$
\begin{aligned}
& \left|f\left(\frac{x+\sigma y}{2}\right)^{2}-g(x) f(y)\right| \leq \psi(x), \\
& \left|f\left(\frac{x+\sigma y}{2}\right)^{2}-g(x) f(y)\right| \leq \psi(y)
\end{aligned}
$$

for all $x, y \in G$, where $\psi: G \rightarrow \mathbb{R}^{+}$is an unbounded function.

Note that the cases $\left|f((x+\sigma y) / 2)^{2}-f(x) g(y)\right| \leq \psi(y)$ and $\left|f((x+\sigma y) / 2)^{2}-f(x) g(y)\right| \leq \psi(x)$ can be reduced to the special case of form (4) or (5) when $\sigma=I$, the identity involution on $G$ (replacing $g(y)$ by $g(\sigma y)$ and $\psi(y)$ by $\psi(\sigma y)$ ).

As a direct consequence of our results, we obtain the following.

Theorem 2. Assume that $f: G \rightarrow \mathbb{C}$ satisfies

$$
\left|f\left(\frac{x+\sigma y}{2}\right)^{2}-f(x) f(y)\right| \leq \delta
$$

for all $x, y \in G$. Then, every bounded function $f$ satisfies

$$
f(x) \leq \frac{m_{f}+\sqrt{m_{f}^{2}+4 \delta}}{2}:=K_{f, \delta}^{*}
$$

for all $x \in G$, and if there exists $y_{0} \in G$ such that $\left|f\left(y_{0}\right)\right|>K_{f, \delta}^{*}$, then there exist $\sigma$-exponential function $m_{\sigma}$ and $\lambda \in \mathbb{C}$ such that

$$
f(x)=\lambda m_{\sigma}(x)
$$

for all $x \in G$.

Remark 3. Let $\sigma=I$ be the identity involution in Theorem 2; we obtain a refined version of Theorem 1 . Now, from (7), we have

$$
\begin{aligned}
M_{f}-m_{f} & \leq \frac{-m_{f}+\sqrt{m_{f}^{2}+4 \delta}}{2} \leq \frac{2 \delta}{m_{f}+\sqrt{m_{f}^{2}+4 \delta}} \\
& \leq \frac{2 \delta}{\sqrt{4 \delta}}=\sqrt{\delta} .
\end{aligned}
$$

Thus, if $\left\{f_{n}\right\}, n=1,2,3, \ldots$, is a sequence of functions satisfying

$$
\left|f_{n}\left(\frac{x+y}{2}\right)^{2}-f_{n}(x) f_{n}(y)\right| \leq \frac{1}{n}
$$

for all $x, y \in G$, then there exists a sequence $r_{n} \in \mathbb{R}$ such that $\left|f_{n}(x)\right|-r_{n}$ converges uniformly to 0 as $n \rightarrow \infty$ (i.e., $\left|f_{n}\right|$ tends to straight lines).

We refer the reader to [5-7] for related functional equations and their stabilities. We also refer the reader to $[8,9]$ for some important recent developments on the issues of stability and superstability for functional equations.

\section{Stability of (4)}

In this section, we prove the superstability of (4). For the proof, we need the following.

Lemma 4. Assume that $m_{1}, m_{2}: G \rightarrow \mathbb{C}$ are unbounded exponential functions satisfying

$$
\left|m_{1}(x)-\lambda m_{2}(x)\right| \leq \delta
$$

for all $x \in G$ and for some $\lambda \in \mathbb{C}$. Then, one has $\lambda=1$ and $m_{1}=m_{2}$.

Proof. Since $m_{2}$ is unbounded exponential function, it is easy to see that $m_{2}(x) \neq 0$ for all $x \in G$. Replacing $x$ by $k x, k=$ $1,2,3, \ldots$, in (11) and dividing the result by $\left|m_{2}(k x)\right|$ we have

$$
\left|\left(\frac{m_{1}(x)}{m_{2}(x)}\right)^{k}-\lambda\right| \leq \frac{\delta}{\left|m_{2}(x)\right|^{k}} .
$$

If $\left|m_{2}(x)\right|>1$, we have

$$
\lim _{k \rightarrow \infty}\left(\frac{m_{1}(x)}{m_{2}(x)}\right)^{k}=\lambda .
$$

Since $\lambda \neq 0$, from (13), we have

$$
m_{1}(x)=m_{2}(x)
$$

for all $x \in G$ such that $\left|m_{2}(x)\right|>1$ and hence $\lambda=1$. Let $\left|m_{2}(x)\right| \leq 1$. Choose $y \in G$ such that $\left|m_{2}(y)\right|>\left|m_{2}(x)\right|^{-1}$. Then, we have $\left|m_{2}(y)\right|>1$ and $\left|m_{2}(x+y)\right|=\left|m_{2}(x) m_{2}(y)\right|>$ 1. Thus, it follows from (14) that $m_{1}(y)=m_{2}(y)$ and $m_{1}(x+$ $y)=m_{2}(x+y)$ and hence

$$
m_{1}(x)=\frac{m_{1}(x+y)}{m_{1}(y)}=\frac{m_{2}(x+y)}{m_{2}(y)}=m_{2}(x)
$$

for all $x \in G$ such that $\left|m_{2}(x)\right| \leq 1$. This completes the proof.

From now on, we denote $M_{f}=\sup _{x \in G}|f(x)|$ and $m_{f}=$ $\inf _{x \in G}|f(x)|$.

Theorem 5. Assume that $f, g: G \rightarrow \mathbb{C}$ satisfy (4). Then, every bounded function $f$ satisfies

$$
M_{f} \leq \inf _{x \in G} \frac{|g(x)|+\sqrt{|g(x)|^{2}+4 \psi(x)}}{2} .
$$

If $f$ is unbounded, then there exists an exponential function $m$ such that

$$
\begin{aligned}
g(x) & =g(0) m(x) \\
|f(x)| & \leq \sqrt{|f(0) g(0) m(2 x)|+\psi(2 x)}
\end{aligned}
$$

for all $x \in G$, and, in particular, if

$$
\inf _{x \in G}\left(\frac{\psi(x)}{|g(x)|}+\frac{1}{|g(x)|}\right)=0
$$


then there exist $\sigma$-exponential function $m_{\sigma}$ and $\lambda \in \mathbb{C}$ such that

$$
f(x)=g(x)=\lambda m_{\sigma}(x)
$$

for all $x \in G$.

Proof. Assume that $f$ is bounded. Replacing $y$ by $-\sigma x+2 \sigma y$ in (4) and using the triangle inequality with the result, we have

$$
\begin{aligned}
|f(y)|^{2} & \leq|g(x)||f(-\sigma x+2 \sigma y)|+\psi(x) \\
& \leq|g(x)| M_{f}+\psi(x)
\end{aligned}
$$

for all $x, y \in G$. Taking the supremum of the left hand side of (20), we have

$$
M_{f}^{2} \leq|g(x)| M_{f}+\psi(x)
$$

for all $x \in G$. Fixing $x$ and solving the quadratic inequality (21), we obtain

$$
M_{f} \leq \frac{|g(x)|+\sqrt{|g(x)|^{2}+4 \psi(x)}}{2}
$$

for all $x \in G$. Taking the infimum of the right hand side of (22), we get (16). Now, assume that $f$ is unbounded. Putting $x=0$ in (4), we have

$$
\left|f\left(\frac{\sigma y}{2}\right)^{2}-g(0) f(y)\right| \leq \psi(0)
$$

for all $y \in G$. Choosing a sequence $y_{n} \in G, n=1,2,3, \ldots$, such that $\left|f\left(y_{n}\right)\right| \rightarrow \infty$ as $n \rightarrow \infty$, putting $y=y_{n}, n=$ $1,2,3, \ldots$, in (4), dividing the result by $\left|f\left(y_{n}\right)\right|$, letting $n \rightarrow \infty$, and using (23), we have

$$
\begin{aligned}
g(x) & =\lim _{n \rightarrow \infty} \frac{f\left(\left(x+\sigma y_{n}\right) / 2\right)^{2}}{f\left(y_{n}\right)} \\
& =\lim _{n \rightarrow \infty} \frac{g(0) f\left(\sigma x+y_{n}\right)}{f\left(y_{n}\right)}
\end{aligned}
$$

for all $x \in G$. Multiplying both sides of (24) by $g(y)$ and using (4) and (24), we have

$$
\begin{aligned}
g(y) g(x) & =\lim _{n \rightarrow \infty} \frac{g(0) g(y) f\left(\sigma x+y_{n}\right)}{f\left(y_{n}\right)} \\
& =\lim _{n \rightarrow \infty} \frac{g(0) f\left(\left(y+x+\sigma y_{n}\right) / 2\right)^{2}}{f\left(y_{n}\right)} \\
& =g(0) g(y+x)
\end{aligned}
$$

for all $x, y \in G$. Thus, $g_{0}(x)=g(x) / g(0)$ is an exponential function and let $g_{0}=m$; then, we get

$$
g(x)=g(0) m(x)
$$

for all $x \in G$. Putting $y=0$ in (4), replacing $x$ by $2 x$, and using (26) and the triangle inequality, we have $|f(x)| \leq$ $\sqrt{\left|f(0) g(0) m(x)^{2}\right|+\psi(2 x)}$ for all $x \in G$, which gives (17). Assume that (18) holds. Then, we can choose a sequence $x_{n}$, $n=1,2,3, \ldots$, such that

$$
\lim _{n \rightarrow \infty} \frac{\psi\left(x_{n}\right)}{\left|g\left(x_{n}\right)\right|}=\lim _{n \rightarrow \infty} \frac{1}{\left|g\left(x_{n}\right)\right|}=0 .
$$

Putting $x=x_{n}, n=1,2,3, \ldots$, in (4), dividing the result by $\left|g\left(x_{n}\right)\right|$, letting $n \rightarrow \infty$, and using (23), we have

$$
\begin{aligned}
f(y) & =\lim _{n \rightarrow \infty} \frac{f\left(\left(x_{n}+\sigma y\right) / 2\right)^{2}}{g\left(x_{n}\right)} \\
& =\lim _{n \rightarrow \infty} \frac{g(0) f\left(\sigma x_{n}+y\right)}{g\left(x_{n}\right)}
\end{aligned}
$$

for all $y \in G$. Multiplying both sides of (28) by $g(x)$ and using (4) and (28), we have

$$
\begin{aligned}
g(x) f(y) & =\lim _{n \rightarrow \infty} \frac{g(0) g(x) f\left(\sigma x_{n}+y\right)}{g\left(x_{n}\right)} \\
& =\lim _{n \rightarrow \infty} \frac{g(0) f\left(\left(x+\sigma y+x_{n}\right) / 2\right)^{2}}{g\left(x_{n}\right)} \\
& =g(0) f(\sigma x+y)
\end{aligned}
$$

for all $x, y \in G$. Putting $y=0$ in (29), replacing $x$ by $\sigma x$, using (26), and dividing the result by $g(0)$, we have

$$
f(x)=f(0) m(\sigma x)
$$

for all $x \in G$. Putting (30) in (23), we have

$$
|f(0)|^{2}\left|m(y)-g(0) f(0)^{-1} m(\sigma y)\right| \leq \psi(0)
$$

for all $y \in G$. Applying Lemma 4 in (31), we have $m(y)=$ $m(\sigma y)$ for all $y \in G$ and $g(0)=f(0)$. Letting $g(0)=f(0):=$ $\lambda$, we get (19). This completes the proof.

In particular, if $g=f$ in Theorem 5, we obtain the following.

Corollary 6. Assume that $f: G \rightarrow \mathbb{C}$ satisfies

$$
\left|f\left(\frac{x+\sigma y}{2}\right)^{2}-f(x) f(y)\right| \leq \psi(x)
$$

for all $x, y \in G$. Then, every bounded function $f$ satisfies

$$
M_{f} \leq \inf _{x \in G} \frac{|f(x)|+\sqrt{|f(x)|^{2}+4 \psi(x)}}{2}
$$

and if $f$ is unbounded, then there exists $\sigma$-exponential function $m_{\sigma}$ such that

$$
f(x)=f(0) m_{\sigma}(x)
$$

for all $x \in G$. 
Proof. By Theorem 5, we get (33) and every unbounded function $f$ has the form $f(x)=f(0) m(x)$ for some exponential function $m$. Putting $x=0$ and $f(y)=f(0) m(y)$ in (32), we have

$$
\left|f(0)^{2}\right||m(\sigma y)-m(y)| \leq \psi(0)
$$

for all $y \in G$. Using Lemma 4, we have $m(y)=m(\sigma y)$ for all $y \in G$. Thus, we get (34). This completes the proof.

Remark 7. In particular, if $\psi(x)=\delta$ in Corollary 6, we obtain Theorem 2.

\section{Stability of (5)}

In this section, we prove the stability of (5). We exclude the trivial case when $f=0$ or $g=0$.

Theorem 8. Assume that $f, g: G \rightarrow \mathbb{C}$ satisfy (5). Then, every bounded function $f$ satisfies

$$
\begin{gathered}
\sup _{y \in G}\left(M_{g}|f(y)|-\psi(y)\right) \leq M_{f}^{2} \\
\leq \inf _{y \in G}\left(M_{g}|f(y)|+\psi(y)\right) .
\end{gathered}
$$

If $f$ is unbounded, then there exists an exponential function $m$ such that

$$
f(x)=f(0) m(x)
$$

for all $x \in G$. In particular, if

$$
\inf _{x \in G}\left(\frac{\psi(x)}{|f(x)|}+\frac{1}{|f(x)|}\right)=0,
$$

then there exist $\sigma$-exponential function $m_{\sigma}$ and $\lambda \in \mathbb{C}$ such that

$$
f(x)=g(x)=\lambda m_{\sigma}(x)
$$

for all $x \in G$.

Proof. Choosing $y_{0} \in G$ such that $f\left(y_{0}\right) \neq 0$ and putting $y=y_{0}$ in (5), we get

$$
\left|f\left(\frac{x+\sigma y_{0}}{2}\right)^{2}-g(x) f\left(y_{0}\right)\right| \leq \psi\left(y_{0}\right)
$$

for all $x \in G$, and it follows that $f$ is bounded if and only if $g$ is bounded. Assume that $f$ and $g$ are bounded. Replacing $x$ by $2 x-\sigma y$ in (5) and using the triangle inequality with the result, we have

$$
\begin{aligned}
|f(x)|^{2} & \leq|g(2 x-\sigma y)||f(y)|+\psi(y) \\
& \leq M_{g}|f(y)|+\psi(y)
\end{aligned}
$$

for all $x, y \in G$. Taking the supremum of the left hand side of (41), we have

$$
M_{f}^{2} \leq M_{g}|f(y)|+\psi(y)
$$

for all $y \in G$. Taking the infimum of the right hand side of (42), we have

$$
M_{f}^{2} \leq \inf _{y \in G}\left(M_{g}|f(y)|+\psi(y)\right) .
$$

Now, using the triangle inequality with (5) again, we have

$$
|g(x) f(y)| \leq\left|f\left(\frac{x+\sigma y}{2}\right)^{2}\right|+\psi(y) \leq M_{f}^{2}+\psi(y)
$$

for all $x, y \in G$. Taking the supremum of $g(x)$ in the left hand side of (44) and subtracting $\psi(y)$ from both sides of the result, we have

$$
M_{g}|f(y)|-\psi(y) \leq M_{f}^{2}
$$

for all $y \in G$. Taking the supremum of the left hand side of (45), we have

$$
\sup _{y \in G}\left(M_{g}|f(y)|-\psi(y)\right) \leq M_{f}^{2} .
$$

From (43) and (46), we get (36). Now, we assume that $f$ and $g$ are unbounded. Putting $y=0$ in (5), we have

$$
\left|f\left(\frac{x}{2}\right)^{2}-g(x) f(0)\right| \leq \psi(0)
$$

for all $x \in G$. Choosing a sequence $x_{n} \in G, n=1,2,3, \ldots$, such that $\left|g\left(x_{n}\right)\right| \rightarrow \infty$ as $n \rightarrow \infty$, putting $x=x_{n}, n=$ $1,2,3, \ldots$, in (5), dividing the result by $\left|g\left(x_{n}\right)\right|$, letting $n \rightarrow \infty$, and using (47), we have

$$
\begin{aligned}
f(y) & =\lim _{n \rightarrow \infty} \frac{f\left(\left(x_{n}+\sigma y\right) / 2\right)^{2}}{g\left(x_{n}\right)} \\
& =\lim _{n \rightarrow \infty} \frac{f(0) g\left(x_{n}+\sigma y\right)}{g\left(x_{n}\right)}
\end{aligned}
$$

for all $y \in G$. Multiplying both sides of (48) by $f(z)$ and using (5) and (48), we have

$$
\begin{aligned}
f(y) f(z) & =\lim _{n \rightarrow \infty} \frac{f(0) g\left(x_{n}+\sigma y\right) f(z)}{g\left(x_{n}\right)} \\
& =\lim _{n \rightarrow \infty} \frac{f(0) f\left(\left(x_{n}+\sigma y+\sigma z\right) / 2\right)^{2}}{g\left(x_{n}\right)} \\
& =f(0) f(y+z)
\end{aligned}
$$

for all $y, z \in G$. Thus, $f_{0}(x)=f(x) / f(0)$ is an exponential function, say $f_{0}=m$; then, we get

$$
f(x)=f(0) m(x)
$$

for all $x \in G$. Assume that (38) holds. Then, we can choose a sequence $y_{n}, n=1,2,3, \ldots$, such that

$$
\lim _{n \rightarrow \infty} \frac{\psi\left(y_{n}\right)}{\left|f\left(y_{n}\right)\right|}=\lim _{n \rightarrow \infty} \frac{1}{\left|f\left(y_{n}\right)\right|}=0 .
$$


Putting $y=y_{n}, n=1,2,3, \ldots$, in (5), dividing the result by $\left|f\left(y_{n}\right)\right|$, letting $n \rightarrow \infty$, and using (47), we have

$$
\begin{aligned}
g(x) & =\lim _{n \rightarrow \infty} \frac{f\left(\left(x+\sigma y_{n}\right) / 2\right)^{2}}{f\left(y_{n}\right)} \\
& =\lim _{n \rightarrow \infty} \frac{f(0) g\left(x+\sigma y_{n}\right)}{f\left(y_{n}\right)}
\end{aligned}
$$

for all $y \in G$. Multiplying both sides of (52) by $f(y)$ and using (5) and (52), we have

$$
\begin{aligned}
g(x) f(y) & =\lim _{n \rightarrow \infty} \frac{f(0) g\left(x+\sigma y_{n}\right) f(y)}{f\left(y_{n}\right)} \\
& =\lim _{n \rightarrow \infty} \frac{f(0) f\left(\left(x+\sigma y_{n}+\sigma y\right) / 2\right)^{2}}{f\left(y_{n}\right)} \\
& =f(0) g(x+\sigma y)
\end{aligned}
$$

for all $x, y \in G$. Putting $x=0$ in (53), replacing $y$ by $\sigma y$, and using (50), we have

$$
g(x)=g(0) m(\sigma x)
$$

for all $x \in G$. Putting (50) and (54) in (47), we have

$$
|f(0)|^{2}\left|m(x)-g(0) f(0)^{-1} m(\sigma x)\right| \leq \psi(0)
$$

for all $x \in G$. Applying Lemma 4, we have $m(x)=m(\sigma x)$ for all $x \in G$ and $g(0)=f(0)$. Letting $g(0)=f(0):=\lambda$, we get (39). This completes the proof. ing.

In particular, if $g=f$ in Theorem 8 , we obtain the follow-

Corollary 9. Assume that $f: G \rightarrow \mathbb{C}$ satisfies

$$
\left|f\left(\frac{x+\sigma y}{2}\right)^{2}-f(x) f(y)\right| \leq \psi(y)
$$

for all $x, y \in G$. Then, every bounded function $f$ satisfies

$$
M_{f} \leq \inf _{x \in G} \frac{|f(x)|+\sqrt{|f(x)|^{2}+4 \psi(x)}}{2},
$$

and if $f$ is unbounded, then there exists $\sigma$-exponential function $m_{\sigma}$ such that

$$
f(x)=f(0) m_{\sigma}(x)
$$

for all $x \in G$.

Proof. By Theorem 8, every bounded function $f$ satisfies

$$
M_{f}^{2} \leq M_{f}|f(y)|+\psi(y)
$$

for all $y \in G$. Solving (59), we have

$$
M_{f} \leq \frac{|f(y)|+\sqrt{|f(y)|^{2}+4 \psi(y)}}{2}
$$

for all $y \in G$, which gives (57). If $f$ is unbounded, then by Theorem 8 we have

$$
f(x)=f(0) m(x)
$$

for all $x \in G$. Putting (61) in (56), we have

$$
\left|f(0)^{2} m(x)\right||m(\sigma y)-m(y)| \leq \psi(y)
$$

for all $x, y \in G$. Since $m$ is unbounded, we have $m(y)=m(\sigma y)$ for all $y \in G$, which gives (58). This completes the proof.

In particular, if $\psi(y)=\delta$ for all $y \in G$ in Theorems 5 and 8 , we obtain the following.

Corollary 10. Assume that $f, g: G \rightarrow \mathbb{C}$ satisfy

$$
\left|f\left(\frac{x+\sigma y}{2}\right)^{2}-g(x) f(y)\right| \leq \delta
$$

for all $x, y \in G$. If $f$ is bounded, then $f$ satisfies

$$
M_{g} M_{f}-\delta \leq M_{f}^{2} \leq \min \left\{M_{g} m_{f}, M_{f} m_{g}\right\}+\delta .
$$

If $f$ is unbounded, then there exist $\sigma$-exponential function $m_{\sigma}$ and $\lambda \in \mathbb{C}$ such that

$$
f(x)=g(x)=\lambda m_{\sigma}(x)
$$

for all $x \in G$.

Proof. By Theorem 5, every bounded function $f$ satisfies

$$
M_{f}^{2} \leq M_{f} m_{g}+\delta
$$

and, by Theorem $8, f$ satisfies

$$
M_{g} M_{f}-\delta \leq M_{f}^{2} \leq M_{g} m_{f}+\delta
$$

From (66) and (67), we get (64). If $f$ is unbounded, then condition (38) holds. Thus, by Theorem 8 , we get (65). This completes the proof.

\section{Examples}

In this section, employing some involutions on Euclidean space, we give various examples satisfying inequalities (4) and (5). We denote by $c \cdot x$ the inner product of $c=\left(c_{1}, c_{2}, \ldots, c_{n}\right) \in$ $\mathbb{C}^{n}$ and $x=\left(x_{1}, x_{2}, \ldots, x_{n}\right) \in \mathbb{R}^{n}$ defined as $c \cdot x=\sum_{j=1}^{n} c_{j} x_{j}$, $|x|=\sqrt{x_{1}^{2}+\cdots+x_{n}^{2}}$, and $\mathfrak{R} c=\left(\Re c_{1}, \ldots, \mathfrak{R} c_{n}\right)$, where $\mathfrak{R}_{c_{j}}$ are the real parts of $c_{j}, j=1,2, \ldots, n$. It is easy to see that if $G$ is uniquely 2-divisible, then $m_{\sigma}$ is $\sigma$-exponential if and only if

$$
m_{\sigma}(x)=m\left(\frac{x+\sigma x}{2}\right), \quad \forall x \in G
$$

for some exponential function $m: G \rightarrow \mathbb{C}$. 
Example 1. Let $G=\left\langle\mathbb{R}^{2},+\right\rangle$ in Theorem 5 and let

$$
\sigma=\left[\begin{array}{cc}
a & b \\
\frac{1-a^{2}}{b} & -a
\end{array}\right]
$$

be a $2 \times 2$ matrix, where $a, b \in \mathbb{R}$ with $b \neq 0$. Then, $\sigma$ defines an involution on $\mathbb{R}^{2}$. Let $f, g: \mathbb{R}^{2} \rightarrow \mathbb{C}$ satisfy

$$
\begin{aligned}
& \mid f\left(\frac{t+a u+b v}{2}, \frac{s+b^{-1}\left(1-a^{2}\right) u-a v}{2}\right)^{2} \\
& \quad-g(t, s) f(u, v) \mid \leq \epsilon\left(|t|^{p}+|s|^{p}\right)
\end{aligned}
$$

for all $t, s, u, v \in \mathbb{R}$ and for some $\epsilon>0, p>0$. Then, by Theorem 5 , every bounded function $f$ satisfies

$$
\begin{aligned}
& |f(u, v)| \\
& \quad \leq \inf _{(t, s) \in \mathbb{R}^{2}} \frac{|g(t, s)|+\sqrt{|g(t, s)|^{2}+4 \epsilon\left(|t|^{p}+|s|^{p}\right)}}{2}
\end{aligned}
$$

for all $u, v \in \mathbb{R}$, and if $f$ is unbounded, then

$$
g(t, s)=g(0,0) m(t, s)
$$

for all $(t, s) \in \mathbb{R}^{2}$, where $m: \mathbb{R}^{2} \rightarrow \mathbb{C}$ is an exponential function. In particular, if $g$ is unbounded, then we can choose $\left(t_{0}, s_{0}\right) \in \mathbb{R}^{2}$ such that $\left|m\left(t_{0}, s_{0}\right)\right|>1$, and hence

$$
\begin{aligned}
& \lim _{n \rightarrow \infty} \frac{\psi\left(n t_{0}, n s_{0}\right)}{\left|g\left(n t_{0}, n s_{0}\right)\right|} \\
& \quad \leq \lim _{n \rightarrow \infty} \frac{\epsilon\left(\left|t_{0}\right|^{p}+\left|s_{0}\right|^{p}\right)}{|g(0,0)|} \lim _{n \rightarrow \infty} \frac{n^{p}}{m\left(t_{0}, s_{0}\right)^{n}}=0,
\end{aligned}
$$

which implies that condition (18) holds. Thus, by Theorem 5 , $g, f$ have the form

$$
g(t, s)=f(t, s)=\lambda m(t, s)
$$

for all $(t, s) \in \mathbb{R}^{2}$, where $\lambda \in \mathbb{C}$ and $m: \mathbb{R}^{2} \rightarrow \mathbb{C}$ is an exponential function satisfying

$$
m(t, s)=m\left(a t+b s, b^{-1}\left(1-a^{2}\right) t-a s\right)
$$

for all $(t, s) \in \mathbb{R}^{2}$. In particular, if $g$ is Lebesgue measurable, $m$ is Lebesgue measurable and has the form $m(t, s)=e^{c_{1} t+c_{2} s}$ for some $c_{1}, c_{2} \in \mathbb{C}$. From condition (75), we have $c_{1}=(1 / b)(1+$ a) $c_{2}$. Thus, $g, f$ has the form

$$
g(t, s)=f(t, s)=\lambda e^{d((1+a) t+b s)}
$$

for all $(t, s) \in \mathbb{R}^{2}$ and for some $d, \lambda \in \mathbb{C}$ with $\mathfrak{R} d \neq 0$.
Example 2. Let $\sigma$ be an $n \times n$ matrix such that $\sigma^{2}=E$, the unit matrix. Suppose that $f, g: \mathbb{R}^{n} \rightarrow \mathbb{C}$ satisfy

$$
\left|f\left(\frac{x+\sigma y}{2}\right)^{2}-g(x) f(y)\right| \leq e^{|x|^{q}}
$$

for all $x, y \in \mathbb{R}^{n}$ and for some $0 \leq q<1$. Then, by Theorem 5 , every bounded function $f$ satisfies

$$
|f(y)| \leq \inf _{x \in \mathbb{R}^{n}} \frac{|g(x)|+\sqrt{|g(x)|^{2}+4 e^{|x|^{q}}}}{2}
$$

for all $y \in \mathbb{R}^{n}$, and if $f$ is unbounded, then

$$
g(x)=g(0) m(x)
$$

for all $x \in \mathbb{R}^{n}$. If $g$ is unbounded, it is easy to see that condition (18) holds. Thus, by Theorem 5 , there exist $\sigma$-exponential function $m_{\sigma}: \mathbb{R}^{n} \rightarrow \mathbb{C}$ and $\lambda \in \mathbb{C}$ such that

$$
g(x)=f(x)=\lambda m_{\sigma}(x)
$$

for all $x \in \mathbb{R}^{n}$. In particular, if $g$ is continuous, then by (68) there exist $c \in \mathbb{C}^{n}, \mathfrak{R}(c+c \sigma) \neq 0$ and $\lambda \in \mathbb{C}$ such that

$$
g(x)=f(x)=\lambda e^{(1 / 2)(c+c \sigma) \cdot x}
$$

for all $x \in \mathbb{R}^{n}$

Example 3. Suppose that $f, g: \mathbb{C} \rightarrow \mathbb{C}$ are unbounded function satisfying

$$
\left|f\left(\frac{z+\bar{w}}{2}\right)^{2}-g(z) f(w)\right| \leq \epsilon|w|^{p}
$$

for all $z, w \in \mathbb{C}$ and for some $p>0$. Then, by Theorem 8, there exists an exponential function $m: \mathbb{C} \rightarrow \mathbb{C}$ such that

$$
f(z)=f(0) m(z)
$$

for all $z \in \mathbb{C}$. Choose $z_{0} \in \mathbb{C}$ such that $\left|m\left(z_{0}\right)\right|>1$. Then, we have

$$
\lim _{n \rightarrow \infty} \frac{\left|n z_{0}\right|^{p}}{\left|f\left(n z_{0}\right)\right|}=\frac{\left|z_{0}\right|^{p}}{|f(0)|} \lim _{n \rightarrow \infty} \frac{n^{p}}{\left|m\left(z_{0}\right)\right|^{n}}=0,
$$

which implies that condition (38) holds. Thus, again, by Theorem 8 , there exist an exponential function $m$ satisfying $m(z)=m(\bar{z})$ and $\lambda \in \mathbb{C}$ such that $f(z)=g(z)=\lambda m(z)$ for all $z \in \mathbb{C}$. Now, from $m(z)=m(\bar{z}), m$ is given by $m(z)=m(\mathfrak{R} z)$. Thus, we have

$$
f(z)=g(z)=\lambda m(\Re z)
$$

for all $z \in \mathbb{C}$.

Remark 11. Let $a, b \in \mathbb{R}^{n}$ be two nonzero vectors that are not parallel; that is, $b \neq r a$ for all $r \in \mathbb{R}$. Then, the hyperplane $b \cdot x=0$ is not parallel to $(b-a) \cdot x=0$ and hence there exists $x_{0} \in \mathbb{R}^{n}$ such that $b \cdot x_{0}>0$ and $(b-a) \cdot x_{0}>0$. If $b=t a$ for some $t \in \mathbb{R}$, then there exists $x_{0} \in \mathbb{R}^{n}$ such that $b \cdot x_{0}>0$ and $(b-a) \cdot x_{0}>0$ if and only if $t>1$. Thus, if $b \neq t a$ for all $t \leq 1$, then there exists $x_{0} \in \mathbb{R}^{n}$ such that $b \cdot x_{0}>0$ and $(b-a) \cdot x_{0}>0$. 
Example 4. Let $a \in \mathbb{R}^{n}$ be fixed. Suppose that $f, g: \mathbb{R}^{n} \rightarrow \mathbb{C}$ are unbounded continuous functions satisfying

$$
\left|f\left(\frac{x+\sigma y}{2}\right)^{2}-g(x) f(y)\right| \leq e^{a \cdot y}
$$

for all $x, y \in \mathbb{R}^{n}$. By Theorem 8 , we have

$$
f(x)=f(0) e^{c \cdot x}
$$

for all $x \in \mathbb{R}^{n}$ and for some $c \in \mathbb{C}^{n}, \lambda \in \mathbb{C}$. If $\Re c \neq t a$ for all $t \leq 1$, then, by Remark 11, there exists $x_{0} \in \mathbb{R}^{n}$ such that

$$
\begin{aligned}
& \Re c \cdot x_{0}>0, \\
& \Re c \cdot x_{0}>a \cdot x_{0} .
\end{aligned}
$$

From (87) and (88), we have

$$
\begin{aligned}
\lim _{k \rightarrow \infty} \frac{1+e^{a \cdot k x_{0}}}{\left|f\left(k x_{0}\right)\right|} & =\frac{1}{|f(0)|} \lim _{k \rightarrow \infty} \frac{1+e^{a \cdot k x_{0}}}{e^{\Re c \cdot k x_{0}}} \\
& =\frac{1}{|f(0)|} \lim _{k \rightarrow \infty} \frac{1+e^{k a \cdot x_{0}}}{e^{k \Re c \cdot x_{0}}}=0
\end{aligned}
$$

Thus, condition (38) holds. By Theorem 8 , we get

$$
g(x)=f(x)=\lambda e^{(1 / 2)(c+c \sigma) \cdot x}
$$

for all $x \in \mathbb{R}^{n}$

\section{Competing Interests}

The authors declare that they have no competing interests.

\section{Acknowledgments}

This research was supported by Basic Science Research Program through the National Research Foundation of Korea (NRF) funded by the Ministry of Education (no. 2015R1D1A3A01019573).

\section{References}

[1] P. Găvrută, "On the stability of some functional equation," in Stability of Mappings of Hyers-Ulam Type, Th. M. Rassias and J. Tabor, Eds., pp. 93-98, Hadronic Press, Palm Harbor, Fla, USA, 1994.

[2] D. H. Hyers, G. Isac, and Th. M. Rassias, Stability of Functional Equations in Several Variables, Birkhäuser, Boston, Mass, USA, 1998.

[3] M. Albert and J. A. Baker, "Bounded solutions of a functional inequality," Canadian Mathematical Bulletin, vol. 25, no. 4, pp. 491-495, 1982.

[4] J. A. Baker, "The stability of the cosine equation," Proceedings of the American Mathematical Society, vol. 80, no. 3, pp. 411-416, 1980.

[5] J. Brzdęk, "On solutions of a generalization of the Reynolds functional equation," Demonstratio Mathematica, vol. 41, no. 4 , pp. 859-868, 2008.
[6] J. Chudziak and J. Tabor, "On the stability of the Gołąb-Schinzel functional equation," Journal of Mathematical Analysis and Applications, vol. 302, no. 1, pp. 196-200, 2005.

[7] A. Najdecki, "On stability of a functional equation connected with the Reynolds operator," Journal of Inequalities and Applications, vol. 2007, Article ID 79816, 3 pages, 2007.

[8] N. Brillouët-Belluot, J. Brzdęk, and K. Ciepliński, "On some recent developments in Ulam's type stability," Abstract and Applied Analysis, vol. 2012, Article ID 716936, 41 pages, 2012.

[9] J. Brzdęk and K. Ciepliński, "Hyperstability and superstability," Abstract and Applied Analysis, vol. 2013, Article ID 401756, 13 pages, 2013. 


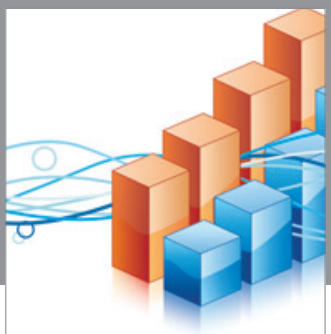

Advances in

Operations Research

vatem alat4

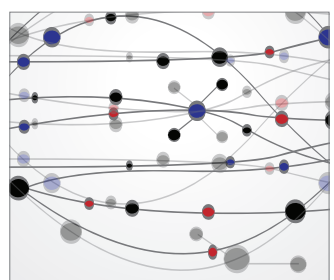

\section{The Scientific} World Journal
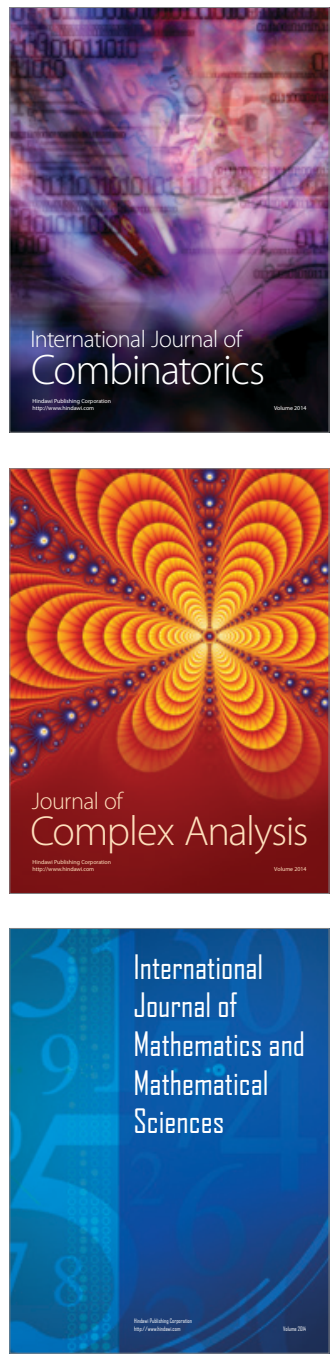
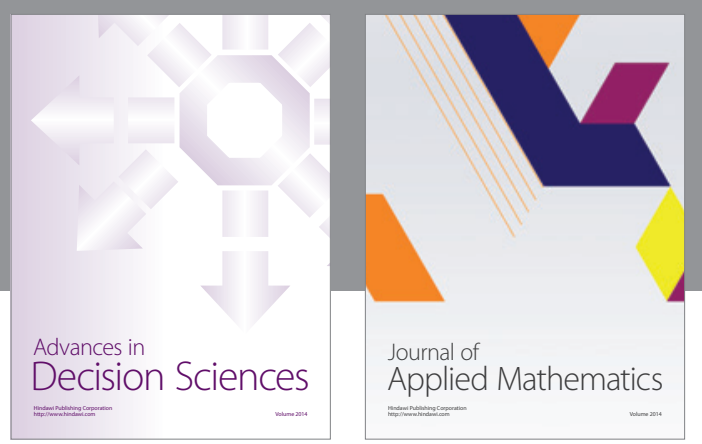

Algebra

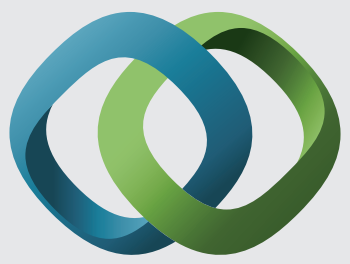

\section{Hindawi}

Submit your manuscripts at

http://www.hindawi.com
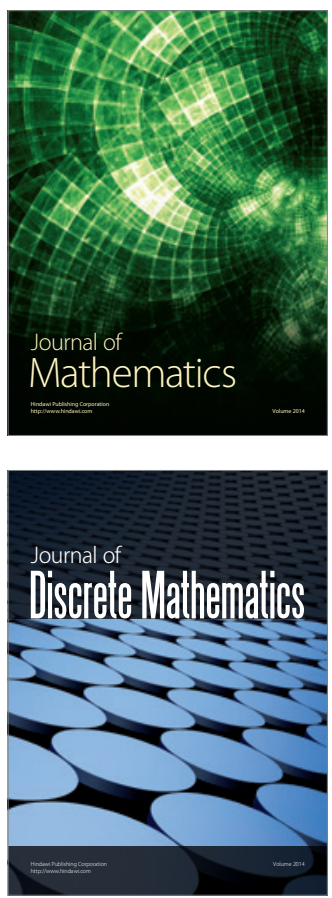

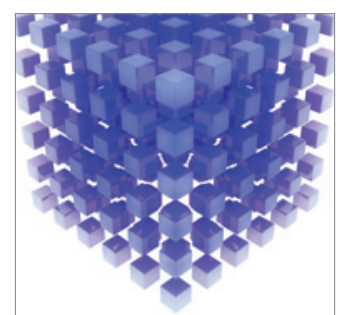

Mathematical Problems in Engineering
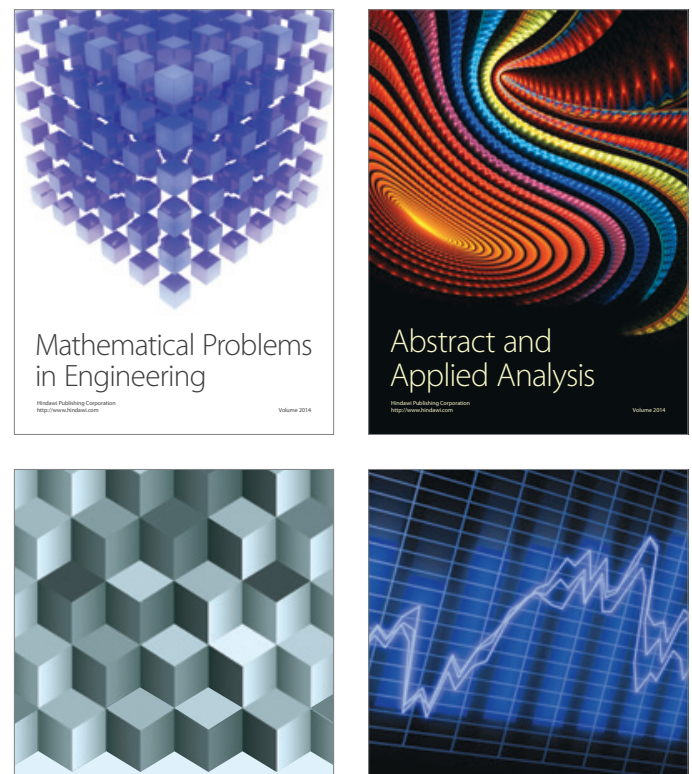

Journal of

Function Spaces

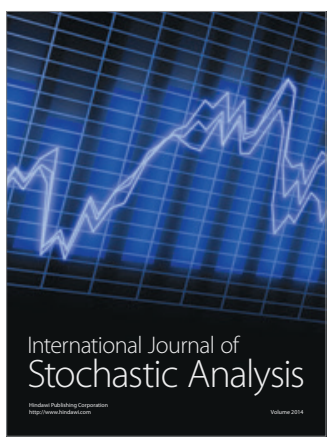

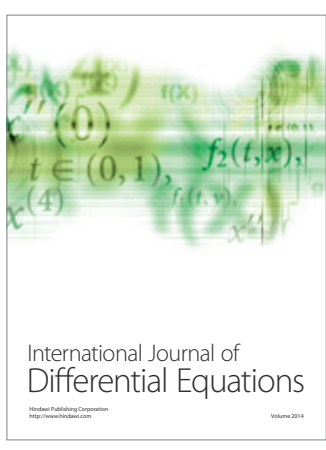
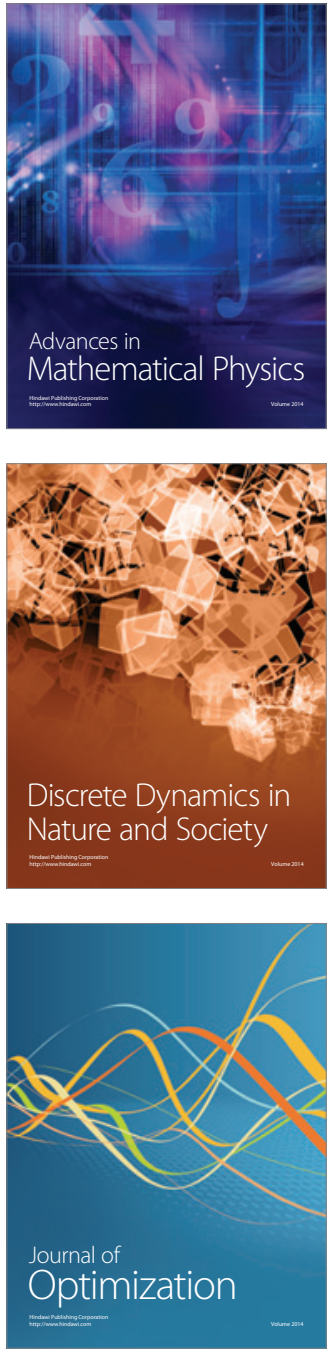